\title{
HD simulations of super star cluster winds
}

\author{
R. Wünsch ${ }^{1}$, J. Palouš ${ }^{1}$, G. Tenorio-Tagle ${ }^{2}$ and S. Silich ${ }^{2}$ \\ ${ }^{1}$ Astronomical Institute, Academy of Sciences of the Czech Republic, Boční II 1401, 14131 \\ Prague, Czech Republic \\ ${ }^{2}$ Instituto Nacional de Astrofísica Optica y Electrónica, AP 51, 72000 Puebla, Mexico
}

\begin{abstract}
We numerically model winds driven by super star clusters (SSC) using the hydrodynamic code ZEUS with the new radiative cooling procedure. The importance of cooling on the wind dynamics depends on the properties of the central cluster: the energy and mass deposition rates $L_{\mathrm{sc}}$ and $\dot{M}_{\mathrm{sc}}$, and the cluster radius $R_{s c}$. Low mass clusters behave adiabatically, and their winds are well described by the solution of Chevalier \& Clegg (1985). However, for larger $L_{\mathrm{sc}}$ and $\dot{M}_{\mathrm{sc}}$ and/or smaller $R_{s c}$, cooling becomes important, and the wind enters the radiative regime in which the wind temperature quickly drops to $10^{4} \mathrm{~K}$ at a small distance away from the cluster (Silich et al., 2004). There is no stationary wind solution for very energetic and compact clusters. This is expressed by the line of the critical luminosity $L_{\text {crit }}$ shown by the left panel as a function of $R_{\mathrm{sc}}$.

In the case of SSC above the threshold line, the stagnation point $R_{\mathrm{st}}$ appears inside the cluster. It splits the cluster volume into two parts: the outer one with $r>R_{\text {st }}$ where the wind velocity is always positive, and the inner one $r<R_{\text {st }}$ where it has a complicated time-dependent profile. The mass inserted into the outer region leaves the cluster in a form of quasi-stationary wind, while most of the mass from the inner region either accumulates there or passes the inner boundary and eventually feeds further star formation. The middle figure shows that the stagnation point $R_{\mathrm{st}}$ asymptotically approaches the cluster radius $R_{\mathrm{sc}}$ with the increasing $L_{\mathrm{sc}}$.

The right figure summarises several of our calculations for a cluster with an $R_{s c}=10 \mathrm{pc}$. It shows the amount of the mass $\dot{M}_{\text {out }}$ outflowing from the cluster depending on $L_{\mathrm{sc}}$. It can be seen that $\dot{M}_{\text {out }}$ grows with $L_{\text {sc }}$ following the power-law fit of the simulations $\dot{M}_{\text {out }} \approx L_{\text {sc }}^{0.54}$. However, the fraction of the outflowing mass to the total mass deposited by the cluster $\dot{M}_{\mathrm{sc}}$ decreases with $L_{\mathrm{sc}}$ from $100 \%$ for $L_{\mathrm{sc}}=L_{\text {crit }}$ to several percent for $L_{\mathrm{sc}}=5 \times 10^{44} \mathrm{erg} \mathrm{s}^{-1}$.
\end{abstract}

Keywords. galaxies: star clusters, galaxies: starburst, ISM: jets and outflows
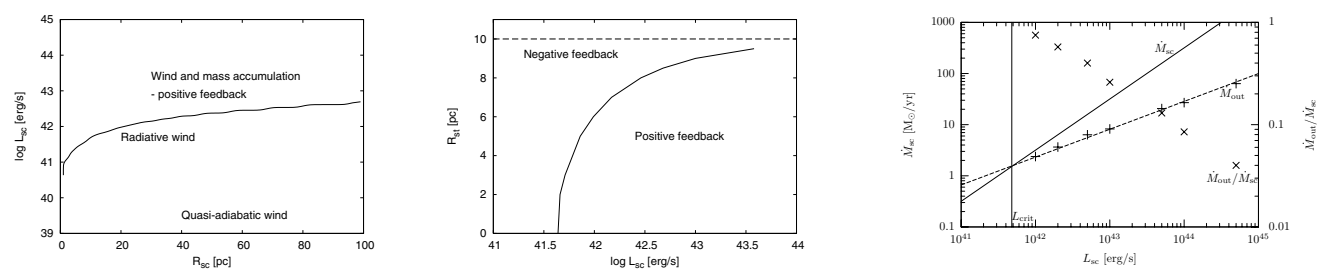

\section{Acknowledgements}

The authors gratefully acknowledge support by the Institutional Research Plan AV0Z10030501 of the Academy of Sciences of the Czech Republic, by the project LC06014 Center for Theoretical Astrophysics, and by CONACyT - México, research grant 47534-F.

\section{References}

Chevalier, R. A. \& Clegg, A. W. 1985, Nature 317, 44

Silich, S., Tenorio-Tagle, G. \& Rodríguez-González, A. 2004, ApJ 610, 226

Tenorio-Tagle, G., Wünsch, R., Silich, S. \& Palouš, J. 2006, in preparation 